\title{
Transport de marchandises et organisation régionale en Hongrie
}

Goods transport and regional organisation in Hungary

Gütertransport und Regionalorganisation in Ungarn

\section{Ferenc Erdosi}

Traducteur : Simon Edelblutte et Emmanuel Chiffre

\section{Q OpenEdition}

\section{Journals}

Édition électronique

URL : http://journals.openedition.org/rge/2454

DOI : $10.4000 /$ rge.2454

ISSN : 2108-6478

Éditeur

Association des géographes de l'Est

Édition imprimée

Date de publication : 1 janvier 2003

ISSN : 0035-3213

\section{Référence électronique}

Ferenc Erdosi, «Transport de marchandises et organisation régionale en Hongrie », Revue

Géographique de l'Est [En ligne], vol. 43 / 1-2 | 2003, mis en ligne le 07 décembre 2010, consulté le 08 septembre 2020. URL : http://journals.openedition.org/rge/2454 ; DOI : https://doi.org/10.4000/rge. 2454

Ce document a été généré automatiquement le 8 septembre 2020

Tous droits réservés 


\title{
Transport de marchandises et organisation régionale en Hongrie
}

\author{
Goods transport and regional organisation in Hungary \\ Gütertransport und Regionalorganisation in Ungarn
}

\section{Ferenc Erdosi}

Traduction : Simon Edelblutte et Emmanuel Chiffre

\section{NOTE DE L'ÉDITEUR}

Cet article a été traduit de l'anglais et adapté par Emmanuel Chiffre et Simon Edelblutte (CERPA / Université Nancy 2)

\section{Introduction}

1 Pays centré sur le bassin des Carpates, vaste plaine développée autour du Danube et de ses affluents, la Hongrie aurait pu posséder un réseau de transport maillé, irriguant de façon homogène ce territoire sans grand relief. Ce n'est pas le cas : le pays présente en effet un réseau en étoile polarisé sur Budapest et dont la densité est plus forte à l'Ouest qu'à l'Est. Cet article, qui ne se veut pas théorique, décrit et analyse, à partir de documents historiques, la mise en place des réseaux de voies de communication et leur impact sur l'organisation et le développement régional. Dans cette perspective géohistorique et nationale, il ne s'intéresse donc peu aux connexions et aux liens avec les pays voisins, en cours de développement aujourd'hui en vue de l'inté gration de la Hongrie à l'Union Européenne.

2 Trois phases chronologiques sont présentées, durant lesquelles les variations très importantes du territoire hongrois, par les fermetures ou ouvertures qu'elles occasionnent, jouent rôle important. La première phase est la formation d'un axe principal danubien et d'axes secondaires sur la Tisza, le Maros et la Drave, tous liés au 
transport de produits agricoles ou du sel. La seconde phase est la plus importante et voit, avec la construction du réseau ferré, une centralisation croissante des réseaux sur Budapest, en raison de multiples facteurs mêlant intérêts politiques (affirmation de la volonté d'indépendance hongroise face à l'Autriche), économiques (volonté des industriels de Budapest d'y faire transiter toutes les marchandises) et considérations techniques (difficulté de franchir le Danube). Cette phase de mise en place du réseau ferré est d'ailleurs aussi à l'origine de mutations importantes au niveau de la hiérarchie urbaine régionale. Enfin, la troisième phase, celle de la construction d'un réseau routier - et surtout autoroutier - moderne, ne fait finalement que confirmer les tendances observées lors de la mise en place du réseau ferré.

\section{La domination du transport fluvial et la formation des axes d'urbanisation}

\section{A. Les premiers temps}

Durant l'Antiquité, la Pannonie, actuelle Transdanubie ${ }^{1}$, fait partie de l'Empire romain ; son réseau routier, relativement bien développé, répond donc à des besoins essentiellement militaires en reliant les places fortes entre elles et à l'Italie. Cependant, les routes, même pavées, ne sont alors pas adaptées au transport en grande quantité de marchandises, pour lequel les voies navigables, essentiellement le Danube et le lac Balaton, sont préférées. Néanmoins, ces flux de marchandises n'ont pas généré l'installation de colonies importantes le long du fleuve ou sur les rives du lac (Dezsényi, Hernády, 1967).

Plus tard, au Moyen Âge, le bétail, exportation hongroise essentielle, est conduit vers les marchés de Vienne et d'Italie. Les chemins qu'utilisent les troupeaux, routes commerciales non pavées vers Buda et empruntées au plus par quelques centaines de négociants et de messagers par an, n'ont cependant pas une importance telle qu'ils puissent modeler la configuration du territoire (Sztankóczy, 1960).

5 Les divisions politiques qui suivent ne favorisent évidemment pas la création d'un réseau routier performant. Ainsi, au XVI ${ }^{\mathrm{e}}$ siècle, la Hongrie est divisée en trois parties, mal reliées entre elles et ne disposant pas, chacune, d'un réseau fiable. Cependant les routes servant au transport du sel vers la Tisza et le Maros existent déjà et, comme les Romains avant eux, les Turcs ottomans considèrent le Danube et la route militaire qui le longe comme les premiers moyens de communication avec Istanbul. Après l'expulsion des Turcs, à la toute fin du XVII ${ }^{e}$ siècle, il faut encore attendre plus d'un siècle avant le début de la modernisation des transports. Il n'y a donc pas, jusqu'au milieu du XIXe siècle, d'évolution majeure, ni dans le réseau et la nature des voies de communication, ni en terme de moyens de transport. Les véhicules tirés par des chevaux sur des routes de terre temporairement praticables constituent donc la règle à cette époque.

\section{B. Une évolution timide au XIXe}

6 Les premières routes pavées sont construites à la fin du XVIII ${ }^{\mathrm{e}}$ siècle à travers le plateau $\mathrm{du}$ Karst et facilitent quelque peu les transports en direction de la Mer Adriatique, de même que s'améliorent les chemins de halage du Danube (Eisenbahn- und 
Dampfschiffarts- Buch, 1852). L'utilisation ancienne et grandissante des voies navigables avait quand même induit des inégalités de peuplement et de développement entre les régions. Cela peut être observé par la formation d'une urbanisation linéaire le long des axes économiques principaux caractérisés par le commerce des produits agricoles, la distribution du sel et le flottage des grumes sur les fleuves. À l'exception des villes du Nord de la Hongrie et de la Transylvanie, nées sur les sources d'énergies et les mines locales, les villes les plus actives de Hongrie, toutes liées au commerce et bénéficiant d'une aire d'approvisionnement suffisante, sont donc situées sur les rives du Danube avec Pozsony (aujourd'hui Bratislava, en Slovaquie), Gyır, Komárom, Vác, Pest-Buda ${ }^{2}$, Dunaföldvár, Paks, Kalocsa, Tolna, Mohács, Zombor (aujourd'hui Sombor, en Serbie), Újvidék (aujourd'hui Novi Sad, en Serbie), mais aussi le long de la Tisza avec Tokaj, Szolnok et Szeged (fig. 1).

Figure 1 : Voies navigables avant la mise en place du réseau ferré

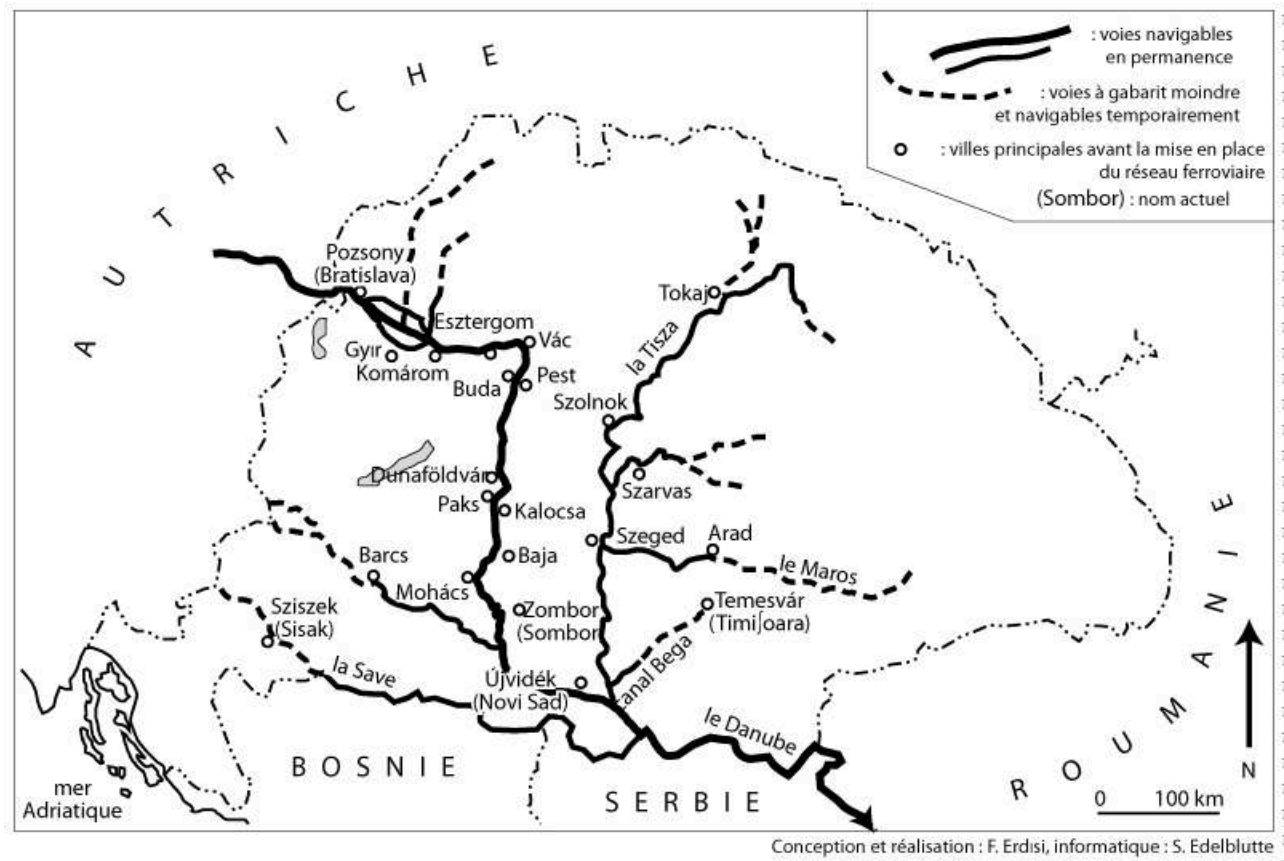

7 La navigation joue également un rôle important dans la croissance rapide de Barcs, Arad (aujourd'hui en Roumanie) et Temesvár (aujourd'hui Timisoara, en Roumanie) respectivement sur la Drave, le Maros Inférieur et le canal Bega. Par ailleurs, et en raison des limites techniques du transport terrestre, d'éventuels liens fondés sur la complémentarité économique des régions n'ont pas pu se développer et se sont cantonnés à de petits territoires, comme les grands domaines fonciers composés de plusieurs villages. Dans ces espaces réduits, les routes, au rôle essentiellement local, ne peuvent donc être utilisées à l'échelle régionale, sauf au prix de très coûteuses améliorations. Le schéma spatial de l'économie et des zones de peuplement consiste ainsi, avant l'arrivée du chemin de fer, en de micro-régions organisées autour de « villes agricoles» espacées en moyenne de 12 à $22 \mathrm{~km}$ les unes des autres (Erdısi, 1996). 


\section{La mise en place d'un réseau ferroviaire très centralisé bouleverse l'organisation spatiale de la Hongrie}

Du milieu du XIX ${ }^{\mathrm{e}}$ siècle à la Première Guerre mondiale, est construit en Hongrie un réseau ferroviaire assez performant, considéré comme en avance sur le niveau socioéconomique général du pays. Par certains côtés, ce réseau est assez semblable à ceux des pays de l'Europe de l'Ouest. Sa mise en place influence beaucoup le développement régional et urbain du pays, à tel point que ces transformations sont encore sensibles aujourd'hui. Ces changements variés et complexes se manifestent d'abord par la naissance d'un réseau très centralisé sur Budapest (fig. 2).

Figure 2 : La mise en place du réseau ferroviaire hongrois de 1867 à 1913

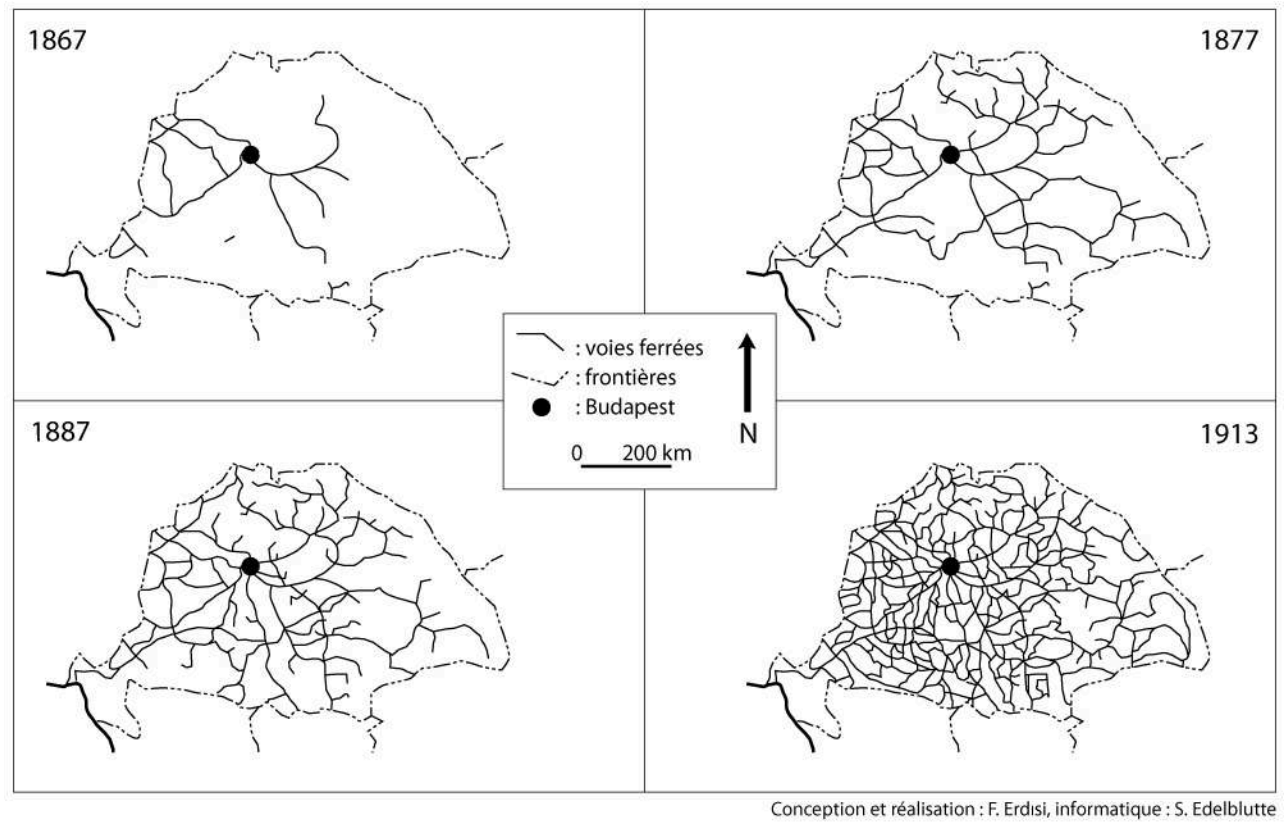

\section{A. Un réseau toujours plus centré sur Budapest}

9 Comme dans plusieurs pays d'Europe - notamment la France - un système de grandes lignes rayonnant à partir de la capitale est adopté, en liaison avec la tendance centralisatrice du pouvoir. En Hongrie cependant, ce processus se singularise de deux manières :

- d'abord par la quasi-absence de lignes transversales, pourtant initialement prévues pour compléter l'étoile créée à partir de Budapest. En France, ces lignes existent, formant un réseau maillé complétant les grandes lignes rayonnant de Paris ;

- ensuite par l'addition aux intérêts centralisateurs de l'État, présents en France également, des intérêts de certains secteurs économiques - notamment industriels - qui ont contribué à l'implantation d'un réseau national centré sur la capitale.

Les efforts pour créer un réseau principal de grandes lignes partant de la capitale apparaissent aussi, dès le début de la construction du réseau, comme une véritable cause nationale. Ils sont d'ailleurs visibles dès 1848 dans le plan du réseau d'István 
Széchenyi, homme d'État hongrois précurseur et parmi les plus en vue à cette époque (Gergely, 1982). L'objectif de cette centralisation est d'améliorer les chances de la Hongrie d'obtenir une indépendance politique dans l'Empire des Habsbourg d'Autriche en permettant à Pest-Buda de rattraper Vienne sur les plans économique et intellectuel, faisant ainsi contrepoids à la capitale autrichienne. Les grandes lignes prévues dans le plan partent donc en étoile de Budapest vers les centres administratifs et économiques hongrois, pour relier, plus tard et au-delà, la Hongrie aux grandes villes des pays voisins (Erdosi, 1986).

11 Parmi ces lignes partant de Pest-Buda, les hommes politiques hongrois accordent une importance particulière à celle qui devait parvenir au port de Fiume (actuelle Rijeka, en Croatie). Ils veulent ainsi contourner une situation nuisible aux intérêts du pays, c'està-dire le passage - fort intéressant pour les Autrichiens - des exportations des produits agricoles hongrois, via le Danube et Vienne, par le port autrichien de Trieste (aujourd'hui en Italie). Fiume (Rijeka), seul grand port hongrois sur la mer Adriatique, offre ainsi la possibilité à la Hongrie de réaliser une politique d'exportations indépendante de celle de l'Autriche.

12 À la fin de la Guerre d'Indépendance de 1848-49, le pouvoir autocratique autrichien tente bien d'affermir les intérêts de l'Empire, souvent opposés à ceux de la nation hongroise, en centrant le réseau ferré de Transdanubie vers Vienne et Trieste. Mais, dans la Grande Plaine Hongroise, bien plus vaste, les lignes construites dans les années 1850 et 1860 partent toutes de Pest $^{3}$, contribuant inévitablement au développement de Pest-Buda. Les Habsbourg ne réussissent donc pas à concrétiser leur projet de tenir les voies ferrées à l'écart de Pest, puisque la ville, pourtant pas la plus peuplée de Hongrie au début du $\mathrm{XIX}^{\mathrm{e}}$ siècle $^{4}$, est quand même devenue le principal centre de commerce du pays, grâce à la navigation sur le Danube et à sa situation au cœur d'une région dynamique. Les raisons de l'échec des Habsbourg sont donc surtout économiques et financières, mais aussi techniques, car il n'était pas possible à l'époque de franchir facilement le Danube et sa large plaine inondable, non encore protégée. Le tableau 1 tente de résumer ces intérêts différents entre le niveau impérial autrichien et le niveau local, via ceux de la Hongrie et des comitats. 
Tableau 1 : Les intérêts territoriaux à différentes échelles et la constitution du réseau ferré hongrois.

\begin{tabular}{|c|c|c|c|c|c|}
\hline $\begin{array}{l}\text { Intérêts territoriaux, } \\
\text { par ordre décroissant }\end{array}$ & $\begin{array}{c}\text { Intérêts impériaux } \\
\text { (officiellement multi- } \\
\text { nationaux, mais en fait } \\
\text { dominés par l'Autriche) }\end{array}$ & $\begin{array}{l}\text { Intérêts nationaux } \\
\text { hongrois }\end{array}$ & Intérêts régionaux & Intérêts provinciaux & Intérêts locaux \\
\hline $\begin{array}{l}\text { Représentants } \\
\text { et défenseurs } \\
\text { de ces intérêts }\end{array}$ & $\begin{array}{c}\text { Gouvernement } \\
\text { de l'Empire d'Autriche } \\
\text { (jusqu'en 1867), } \\
\text { le gouvernement commun } \\
\text { de la Double-Monarchie } \\
\text { (apres 1807), } \\
\text { par lintermédiaire } \\
\text { du Ministere } \\
\text { des Affaires Militaires }\end{array}$ & $\begin{array}{c}\text { Nation, société } \\
\text { et intérêts économiques } \\
\text { hongrois avant } 1867, \\
\text { Parlement et gouvemement } \\
\text { hongrois aprés } 1867 \\
\text { (Ministère des Transports, } \\
\text { puis Ministère du Commerce) }\end{array}$ & $\begin{array}{l}\text { Chambres Régionales } \\
\text { du Commerce } \\
\text { et de l'Industrie }\end{array}$ & Autorités des Comitats & $\begin{array}{l}\text { Autorités municipales } \\
\text { des villes, de villages, } \\
\text { grands proprietaires, } \\
\text { industriels, particuliers }\end{array}$ \\
\hline $\begin{array}{l}\text { Principaux moyens } \\
\text { d'action de ces intérêts }\end{array}$ & $\begin{array}{c}\text { Plan d'un réseau } \\
\text { ferré impérial, } \\
\text { dans un cadre public } \\
\text { (jusqu'en 1854), } \\
\text { puis privé (1854-1867) } \\
\text { sous linfuuence de l'́tat, } \\
\text { le tout supportét } \\
\text { par des moyens financiers } \\
\text { et légaux }\end{array}$ & $\begin{array}{c}\text { Dans un système } \\
\text { de propriété mixte, } \\
\text { puis publique, } \\
\text { construction de lignes } \\
\text { concédées par I'tetat, } \\
\text { nationalisation de certaines } \\
\text { et accords d'exploitation } \\
\text { avec les lignes privées } \\
\text { restantes. Mis en ouvree } \\
\text { par des moyens financiers } \\
\text { et légaux }\end{array}$ & $\begin{array}{c}\text { En rassemblant et } \\
\text { en analysant les donnnées } \\
\text { sur le transport, } \\
\text { en relevant les défauts } \\
\text { du réseau, émet } \\
\text { des suggestions } \\
\text { pour la construction } \\
\text { de nouvelles lignes } \\
\text { et les fait connaitre } \\
\text { par des moyens } \\
\text { de lobbying et de publicité }\end{array}$ & $\begin{array}{c}\text { L'action des comitats } \\
\text { varie d'un comitat à l'autre. } \\
\text { Elle s'effectue par } \\
\text { des questions au Parlement, } \\
\text { des campagnes de presse, } \\
\text { relayés par } \\
\text { des moyens financiers, } \\
\text { des décrets locaux } \\
\text { et de la publicité }\end{array}$ & \begin{tabular}{|c|} 
Laction des villes, villages \\
et des particuliers \\
est évidemment très \\
varíé \\
et s'effectue par \\
des questions au \\
Parlement, \\
des campagnes de presse, \\
relayés par \\
des moyens financiers, \\
des décrets locaux \\
et de la publicité
\end{tabular} \\
\hline $\begin{array}{l}\text { Types de lignes } \\
\text { mises en place } \\
\text { par ces intérêts }\end{array}$ & \multicolumn{2}{|c|}{$\begin{array}{l}\text { Voies ferrées principales } \\
\text { (construites pour l'essentiel entre } 1846 \text { et } 1883 \text { ) }\end{array}$} & \multicolumn{3}{|c|}{$\begin{array}{l}\text { Voies ferrées secondaires, d'intééết local } \\
\text { (principalement construites entre } 1880 \text { et 1914) }\end{array}$} \\
\hline $\begin{array}{l}\text { Principal objectif } \\
\text { poursuivi par ces intérêts } \\
\text { dans la mise en place } \\
\text { du réseau ferré }\end{array}$ & $\begin{array}{c}\text { Un réseau centré } \\
\text { sur Vienne et Trieste, } \\
\text { négligeant quelque peu } \\
\text { Pest-Buda et avec } \\
\text { de rares connections } \\
\text { vers le réseau } \\
\text { du Sud-Est européen }\end{array}$ & 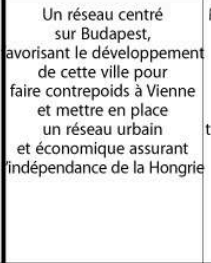 & $\begin{array}{c}\text { Mise en place d'un réseau } \\
\text { de lignes secondaires } \\
\text { favorisant } \\
\text { le trafic interrégional } \\
\text { sans utiliser } \\
\text { les lignes principales } \\
\text { trop chères et encombrées }\end{array}$ & \begin{tabular}{|c|} 
Favorise la mise en place \\
de lignes radiales \\
à partir des cheff-lieux \\
de comitats \\
pour les développer et \\
etendre leur aire dinfluence. \\
Connexion de \\
ces réseaux locaux \\
aux lignes principales \\
vers la capitale, \\
via le chef-lieu du comitat
\end{tabular} & \begin{tabular}{|c|} 
La gare doit être \\
le plus près possible \\
de la ville ou du village. \\
Les grands propriétaires \\
cherchent à sássurer \\
des facilités \\
de chargement \\
dans les evirirons de \\
leurs entrepots céréaliers, \\
sur une ligne \\
ralliant rapidement \\
le marché urbain \\
le plus proche
\end{tabular} \\
\hline $\begin{array}{l}\text { Réalisation } \\
\text { de ces objectifs }\end{array}$ & $\begin{array}{l}\text { Objectifs rarement et } \\
\text { très ponctuellement réalisé }\end{array}$ & $\begin{array}{l}\text { Objectifs totalement } \\
\text { réalisés à la charnière } \\
\text { des XIXe et XXe siècles }\end{array}$ & Objectifs rarement réalisés & $\begin{array}{c}\text { Objectifs réalisés } \\
\text { dans la plupart des comitats }\end{array}$ & $\begin{array}{l}\text { Objectifs } \\
\text { fréquemment réalisés }\end{array}$ \\
\hline
\end{tabular}

Les barres verticales plus épaisses signifient une forte opposition d'intérêts.

Le réseau radial, construit au tournant des années 1860 et 1870 à partir de Budapest, relie donc la capitale à l'Autriche (Vienne, Graz et Trieste), mais aussi à la Transylvanie, à la Haute Hongrie ${ }^{5}$ et à la Subcarpartie ${ }^{6}$. Plus tard, au milieu des années 1870, ce réseau est étendu à la Croatie, alors rattachée au Royaume de Hongrie, vers la Galicie, à la Bucovine et à la Silésie tchèque, appartenant toutes à l'Autriche, et, enfin, par les cols et défilés des Carpates, vers les provinces roumaines voisines de la Transylvanie (Erdosi, 1990). Renforçant encore la centralisation, les minotiers hongrois et leur lobby, le plus puissant d'Europe derrière celui de Rotterdam, font parvenir à Pest, par voies fluviale ou ferrée, les céréales de la Grande Plaine Hongroise. Mieux encore, et afin de capter les productions céréalières des zones frontalières du royaume, plus lointaines, des tarifs ferroviaires préférentiels et différenciés sont mis en place. Ainsi, une bonne partie des produits céréaliers hongrois peut être exportée par Fiume (Rijeka) à des prix extrêmement bas, concurrençant les productions de l'Europe occidentale. L'industrie minotière, qui domine alors la structure industrielle de la capitale, contribue donc au développement de Budapest (Erdosi, 1985).

\section{B. Les tentatives de lutte contre la centralisation du réseau}

Les grands propriétaires céréaliers du Sud et de l'Est de la Grande Plaine Hongroise ne peuvent cependant pas accepter que la capitale, en tant qu'intermédiaire obligé, recueille l'essentiel du profit du commerce céréalier. Ils s'associent donc pour construire une ligne transversale qui doit leur ouvrir un accès direct au port d'exportation de Fiume (Rijeka) par un raccourci évitant Budapest (fig. 3). Au tournant 
des années 1860-1870, une première section de la ligne ferroviaire "Grande Plaine Hongroise - Fiume (Rijeka)» est construite, reliant Nagyvárad (aujourd'hui Oradea, en Roumanie) à Eszék (aujourd'hui Osijek, en Croatie) via Békéscsaba, Szeged, Szabadka (aujourd'hui Subotica, en Serbie) et Zombor (aujourd'hui Sombor, en Serbie). Cependant, le renforcement de la politique centralisatrice des transports met un coup d'arrêt aux travaux de la voie en direction de Fiume (Rijeka) par la Slavonie. La construction d'une autre ligne transversale, nommée Danube - Drave, est alors entreprise, reliant Gyékényes, sur la voie Budapest - Fiume (Rijeka), à Szabadka (Subotica), sur la ligne construite précédemment, via Kaposvár et Dombóvár (fig. 3). Elle est cependant interrompue en 1873, avant d'atteindre le Danube, dont le franchissement pose alors de gros problèmes.

Figure 3 : La ligne Nagyvárad-Fiume et les modifications de l'organisation régionale

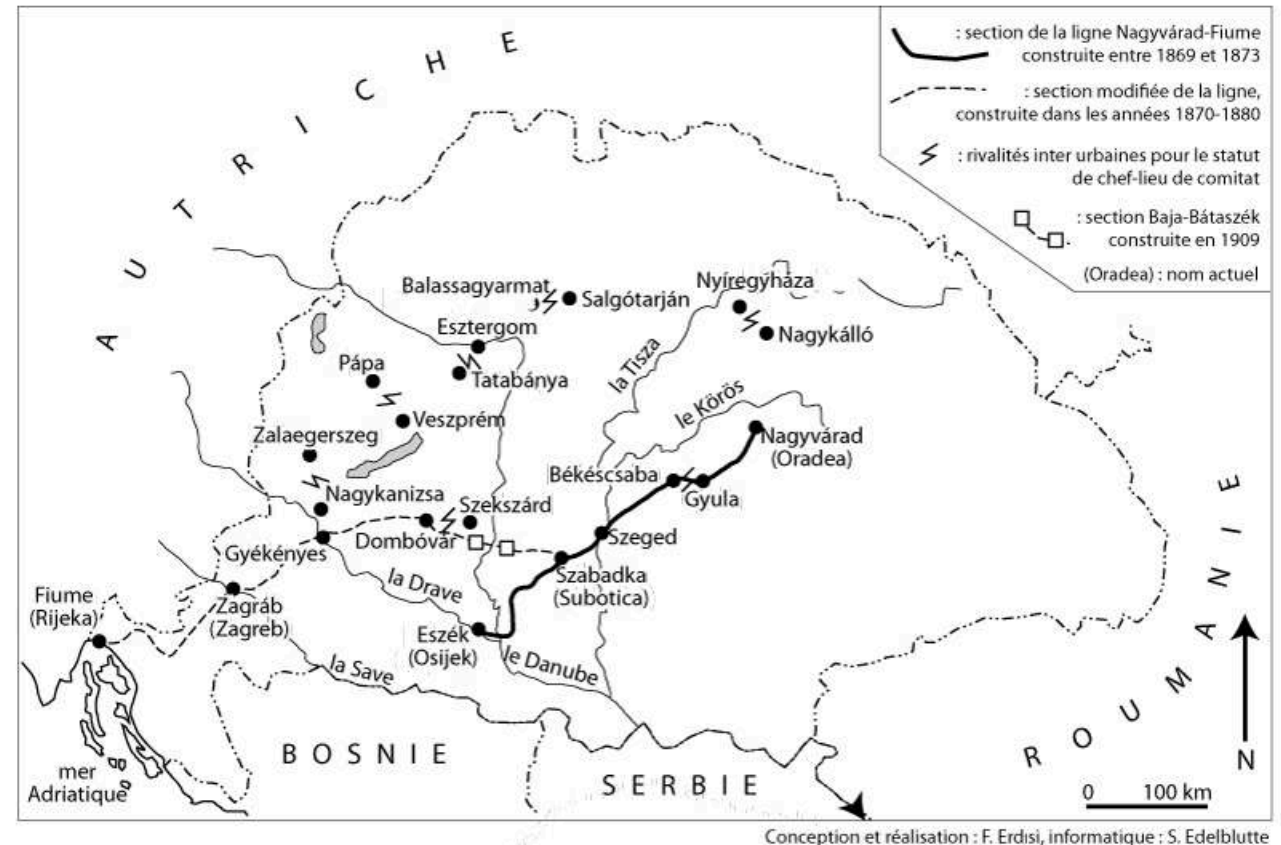

Dans le même temps, la Compagnie des Chemins de Fer Hongrois continue à construire d'autres lignes radiales à partir des années 1880 : d'abord une section de la ligne trans-européenne en direction de Salonique et d'Istanbul, reliant Budapest à Zimony (aujourd'hui Zemun, en Serbie) sur la frontière serbe, tout près de Belgrade ; puis une ligne vers Pécs en Transdanubie du Sud et, plus tard, une ligne vers Tapolca à proximité de la rive Nord du Lac Balaton. D'autres lignes, construites dans la Grande Plaine Hongroise et dans l'extrême Nord du pays, consolident davantage la position de Budapest (Erdosi, 1987a) et il faut attendre finalement 36 ans avant que la ligne transversale méridionale soit terminée avec la construction du pont sur le Danube et des $19 \mathrm{~km}$ manquants entre Bátaszék et Baja (fig. 3).

Ce projet ne s'est finalement concrétisé que sous la pression des groupes d'intérêt économique des régions frontalières méridionales, qui ont mené une campagne active auprès du gouvernement pour l'établissement de liens directs avec la Mer Adriatique. De plus, au début du XXe siècle, les gares de Budapest sont de plus en plus surchargées et l'industrie minotière, face à la diversification du tissu industriel budapestois, a perdu sa position dominante. Ainsi, les autorités acceptent-elles finalement, en 1909, la réalisation de ce lien entre la Transdanubie méridionale et la Grande Plaine Hongroise du 
Sud. Après une amélioration de la ligne en 1914, les trains rapides commencent enfin à circuler sur cette longue transversale entre Nagyvárad (Oradea) et Fiume (Rijeka). En réalité, cette importante réalisation n'a aucun impact sur l'organisation régionale hongroise, puisque la voie est rapidement interrompue en de nombreux endroits par les frontières des nouveaux États issus de la décomposition de l'Empire d'AutricheHongrie après la Première Guerre mondiale. La circulation ne peut donc plus, après la guerre, se faire sur l'intégrité de la ligne ; de petites sections sont même détruites.

17 Le réseau ferré - et par la-même l'organisation régionale - de la Hongrie devient donc plus centralisé à partir des années 1920 ; cette tendance est encore renforcée pendant l'Entre-deux-guerres par la construction de grandes lignes de Budapest à Szeged, Nagykanizsa, Debrecen pour les principales (Erdosi, 1988).

\section{Réseau ferroviaire et réseau urbain}

Les changements de l'organisation spatiale provoqués par la mise en place du réseau ferroviaire se manifestent non seulement par le renforcement de la centralisation du pays, mais aussi par l'affirmation, au sein des villes de province, de centres urbains mieux reliés au réseau que d'autres et devenant de véritables «noyaux de cristallisation » concentrant le dynamisme (fig. 3).

\section{A. Le rôle de la voie ferrée dans le développement urbain à l'échelle nationale}

L'impact des chemins de fer sur le réseau urbain provincial est plus marqué en Hongrie que dans les pays d'Europe Occidentale. Les régions desservies par les gares, regroupant environ un tiers des villes et villages de Hongrie, sont évidemment favorisées. Ce nouveau mode de transport, plus efficace que les anciens, devient donc le vecteur du développement économique ; il entraîne la croissance rapide d'un certain nombre de villes et de villages se trouvant sur les embranchements ou carrefours ferroviaires. Ces derniers constituent de plus une puissante force d'attraction pour les industries à forte demande de transport (briqueteries, minoteries) mais également pour les mines de charbon et les carrières dans les secteurs où ces ressources naturelles sont présentes. Cependant, et à l'inverse de l'Europe de l'Ouest densément peuplée et plus précocement et massivement industrialisée, le niveau plus faible du développement industriel et de la densité de population en Hongrie, ne permet pas à chaque embranchement de devenir une ville ou un centre industriel important. En effet, les embranchements situés en milieu rural, mais à proximité des villes principales et notamment des chefs-lieux de comitats, peuvent difficilement se développer, en raison de la concurrence de la ville plus ancienne, au-delà du niveau induit par la seule présence de la fonction de transport. Ces implantations deviennent donc de simples "villes ferroviaires", regroupement d'employés des chemins de fer, à peine plus développés que les bourgs ruraux environnants. Les chances de devenir des centres économiques et culturels sont en fait bien meilleures pour les embranchements situés en milieu pleinement rural - loin des chefs-lieux - ou implantés dans de petites villes déjà constituées (Erdosi, 1989). 

certaines villes aux dépens d'autres, mais la capacité des différentes lignes a également contribué à cette différenciation entre les villes de province. En effet, les grandes lignes établies dans la première période de la construction jusqu'aux années 1880 jouent un rôle plus important dans le développement des structures économiques, urbaines et régionales que les lignes secondaires construites plus tard. Ces lignes, permettant à l'époque un trafic à longue distance et international, préfigurent les axes et couloirs de développement à l'échelle régionale et nationale toujours visibles aujourd'hui. Elles ont ainsi concentré l'essentiel des forces économiques et urbaines de la Hongrie.

21 le chemin de fer, sont toujours, malgré la concurrence du réseau routier, les éléments principaux et structurants de l'organisation spatiale globale du pays. La précocité de l'équipement ferroviaire constitue donc un avantage spatial décisif pour des villes comme Székesfehérvár, Gyor, Szombathely et Nagykanizsa en Transdanubie ou Cegléd, Kecskemét, Szeged, Debrecen et Nyíregyhása dans la Grande Plaine Hongroise. l'échelle régionale, à des compromis entre les différents intérêts locaux. Ainsi, dans certains cas, les embranchements et les carrefours du réseau sont placés non pas dans les chefs-lieux mais dans d'autres villes. Par conséquent, ces villes bénéficiant d'un embranchement ou d'un carrefour ferroviaire se développent plus rapidement sur le plan économique que les chefs-lieux traditionnels des comitats... Certains acquièrent même le statut de chef-lieu, devenant le centre administratif du comitat aux dépens de l'ancien chef-lieu déclassé. C'est ainsi que, dans le comitat de Békés ${ }^{7}$, le chef-lieu passe de Gyula à Békéscsaba, dans le comitat de Nógrád, Salgótarján supplante Balassagyarmat et, enfin, dans le comitat de Komárom-Esztergom, Tatabánya remplace Esztergom (fig. 3). Dans ces deux derniers cas cependant, le développement croissant des mines de charbon et de l'industrie lourde ont largement contribué à cette migration administrative.

\section{B. Le réseau ferré secondaire et l'organisation spatiale}

Contrairement au réseau des grandes lignes, modelé en fonction d'intérêts nationaux, le réseau de lignes secondaires, établi dans la deuxième phase de construction, à partir de 1880, répond essentiellement à des intérêts régionaux. Ces lignes jouent ainsi un rôle important en reliant les zones non encore desservies aux grandes lignes du réseau de transport. Néanmoins, en raison du faible développement du capitalisme hongrois de l'époque, elles n'attirent pas les industries et leur impact sur la croissance des villes voisines est très faible, à l'exception des régions minières. Elles ne pouvaient en effet pas rivaliser avec les grandes lignes en raison de coûts de transport plus élevés et du faible développement des zones desservies et elles sont donc incapables d'engendrer d'elles-mêmes un processus de développement. Ces voies d'intérêt local reliant les zones agricoles au réseau de transport jouent néanmoins un rôle indirect dans le développement régional et l'urbanisation. L'intensité de cet impact varie cependant d'un comitat à l'autre, en raison des circonstances de leur création et/ou de facteurs historiques.

Dans les comitats dont les chefs-lieux sont devenus des villes relativement importantes dès le début de la construction des voies ferrées (Gyor, Baranya, Somogy, Fejér, Szolnok,

Revue Géographique de l'Est, vol. 43 / 1-2 | 2003 
Hajdú pour les principaux), la plus grande partie du réseau d'intérêt local rayonne à partir de ces pôles. Les autorités locales s'efforcent de plus de promouvoir les lignes secondaires afin qu'elles soient un facteur dominant du développement de leur cheflieu; elles œuvrent donc pour que les voies partent de ce dernier. Parallèlement, les chefs-lieux craignent la construction de lignes d'intérêt local à la périphérie de leur comitat, car ces voies risquent de relier une partie de leur territoire avec des villes de comitats voisins, ou encore peuvent détourner une partie du trafic du chef-lieu du comitat vers des centres secondaires.

La situation est différente dans les comitats où les grandes lignes ne passent pas par le chef-lieu et où d'autres villes ont capté les flux et sont par là même devenues les principaux centres économiques. Cette double centralité (fig. 3), encore perceptible dans les comitats de Veszprém (Veszprém et Pápa), Zala (Zalaegerszeg et Nagykanizsa) et Tolna (Szekszárd et Dombóvár), a certes pour origine essentielle la mise en place du réseau ferroviaire national, mais elle est encore renforcée par la construction des lignes secondaires. En effet, dans ces comitats et à l'inverse des comitats où le chef-lieu est le seul centre sur le plan des transports et de l'économie, le réseau de lignes secondaires n'est pas concentré sur le chef-lieu (Szekszárd pour le comitat de Tolna), ou très peu (Veszprém pour le comitat éponyme et Zalaegerszeg pour le comitat de Zala). Par ailleurs, les centres secondaires nés sur des carrefours ferroviaires à l'époque de la construction des grandes lignes, ne réussissent pas non plus à concentrer les lignes secondaires comme Nagykanizsa (Zala), ou très difficilement comme Pápa (Veszprém) et Dombóvár (Tolna). Le réseau de lignes secondaires n'est donc polarisé ni par les chef-lieux, ni par les centres économiques.

Certes, d'autres facteurs interviennent dans le développement de telle ou telle ville, chef-lieu ou non. Cependant, il reste indiscutable que Zalaegerszeg, devenu un carrefour ferroviaire mineur, n’a pas connu le développement escompté. Inversement, Kaposvár se développe beaucoup après les années 1890, lorsque les lignes secondaires viennent s'y raccorder à la ligne principale, tandis que le développement de Nagykanizsa s'interrompt brutalement avec l'arrêt de leur construction (Erdosi, 1987b).

La mise en place du réseau ferré hongrois a donc joué un rôle fondamental dans la construction du territoire national et des territoires locaux. Les réseaux routier et autoroutier ont-ils joué un rôle comparable?

\section{Les changements à l'ère de l'automobile}

La route et l'autoroute (fig. 4), spécialement adaptées au transport de petites quantités de marchandises sur de courtes distances, n'ont pas fondamentalement modifié l'organisation spatiale liée au réseau ferré (Unyi, 1989). Néanmoins, l'utilisation massive des camions et des automobiles et la construction d'infrastructures routières ont tout de même un impact important au niveau local et plus particulièrement urbain. 
Figure 4 : Le réseau de voies rapides en Hongrie au 1er janvier 2003

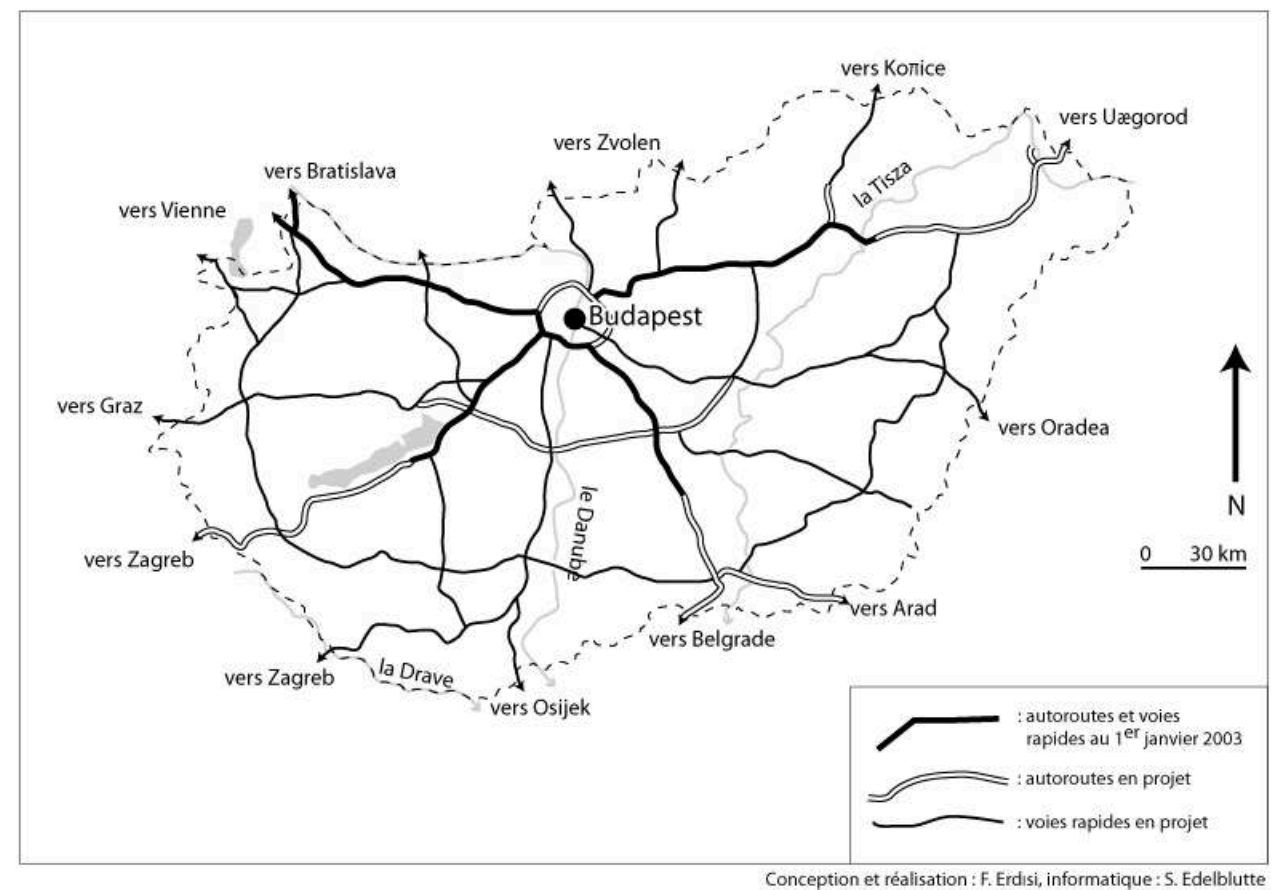

\section{A. Le poids des intérêts locaux dans le tracé des routes}

À l'inverse de celui des voies ferrées, le tracé et la direction des routes principales n'ont fait l'objet de plans que pour un petit nombre. Ces routes remontent en effet à des temps si anciens que leur origine a souvent été oubliée, à l'exception de quelques routes internationales. Par contre, la construction de routes secondaires revêtues, remplaçant les anciens chemins de terre, est le plus souvent planifiée par divers intérêts locaux qui décident ainsi de leur largeur, du type de revêtement et ainsi de leur importance future (Erdosi, 2000).

Cette tendance à laisser faire les intérêts locaux est caractéristique de la Hongrie où les circonscriptions régionales ont souvent été considérées comme seules responsables du développement économique local. Ces choix ont donc fréquemment été opérés sans concertation avec le pouvoir central ou sans coopération entre les différentes circonscriptions administratives. Cette sorte de politique isolationniste ou autarcique est particulièrement sensible au niveau essentiel des comitats où les chefs-lieux, à quelques exceptions près, constituent les centres économiques les plus importants de leur territoire. Dans chaque comitat, le réseau routier secondaire est donc marqué par une polarisation assez forte autour du chef-lieu ; les autres villes n'ayant généré, au mieux, que des carrefours routiers mineurs (Vásárhelyi, 1959).

\section{B. - Un réseau autoroutier polarisé par Budapest}

La fonction principale des autoroutes est d'assurer des liaisons rapides entre les grandes agglomérations et les centres économiques. Le choix de leur tracé est donc effectué, au contraire des routes évoquées ci-dessus, en fonction de la tradition centralisatrice historique. Il suit ainsi celui des voies ferrées principales, les longeant 
le plus souvent et, malgré les critiques dénonçant une centralisation excessive, il est prévu de conserver pour les prochaines réalisations cette structure radiale traditionnelle (Erdosi, 1987c).

Construites très irrégulièrement à partir des années 1960, les autoroutes, se conformant au plan gouvernemental de développement des transports à moyen terme, rendent donc encore plus spectaculaire l'organisation en étoile du réseau de communication déterminée par les lignes ferroviaires. Mais alors qu'elles n'apportent pratiquement aucun changement à la structure régionale à petite échelle, elles tiennent lieu, à grande échelle, d'axes de localisation particulièrement puissants car, contrairement à celles de l'ère socialiste, les sociétés internationales actuelles tiennent compte de la proximité des autoroutes pour s'implanter. Ainsi, autour de Budapest et des grandes villes de province, les autoroutes attirent-elles des services (centres commerciaux, restaurants, concessionnaires automobiles, stations-service, hôtels) et dans certains cas des usines, constituant ainsi des éléments essentiels de la morphologie urbaine. Par l'attrait qu'elles exercent sur les activités économiques, les autoroutes construites récemment - principalement en Transdanubie du Nord augmentent encore les chances pour cette région de Hongrie, déjà la plus avancée, de se développer toujours plus rapidement que le reste du pays, en particulier par rapport aux régions de l'Est, creusant davantage les différences régionales sur le plan du développement.

Cependant, la première bénéficiaire des constructions d'autoroutes reste Budapest, d'abord parce qu'elle est reliée sans discontinuité à l'Europe de l'Ouest, mais aussi parce qu'elle a des liaisons autoroutières directes avec la région touristique la plus importante du pays, le lac Balaton. La capitale a de plus réussi à renforcer son influence sur la Grande Plaine Hongroise grâce aux autoroutes du Nord-Est et du Sud-Est, aujourd'hui longues respectivement de 80 et $100 \mathrm{~km}$ (fig. 4). Bien que les discours politiques - souvent contredits par les réalisations - placent, depuis des décennies, les transports au centre du développement régional, les réalisations des cinq dernières années montrent seulement deux importants groupes de réalisations, sur des points d'ailleurs opposés. Ainsi, à petite échelle, les liaisons internationales, notamment en direction de l'Ouest européen, ont été améliorées et, à grande échelle, chaque village a été relié au réseau routier national et est aujourd'hui desservi par autobus. Par contre, pratiquement aucune création de grandes liaisons interrégionales ferroviaire ou routière - évitant Budapest n'a été réalisée, en particulier entre le Sud de la Grande Plaine Hongroise et le Sud de la Transdanubie (Erdısi, 1992).

\section{Conclusion}

Deux idées majeures se dégagent de cette étude des liens entre voies de communication et organisation régionale de la Hongrie :

- tout d'abord, la centralisation très forte des réseaux ferré et routier, à échelle nationale, comme, dans une moindre mesure cependant, à échelle régionale. La capitale, mais la plupart des chef-lieux de comitat, ont ainsi polarisé les axes pour des raisons à la fois géographiques - vaste plaine développée autour du Danube - historiques - réduction des deux-tiers du territoire hongrois après le Traité de Trianon en 1920 - politiques volonté de renforcer Budapest face à Vienne - techniques et financières - difficulté de franchir le Danube à l'aval de Budapest. L'échec des tentatives, pourtant récurrentes depuis 
le XIX $\mathrm{X}^{\mathrm{e}}$ siècle, de construire des lignes ou des autoroutes ne passant pas par Budapest montre le poids considérable de la centralisation dans un pays où la capitale regroupe aujourd'hui un cinquième des habitants ;

- ensuite, le déséquilibre régional entre l'est et l'ouest du pays apparaît durant chaque phase de la mise en place des réseaux et ce, dès l'époque romaine... L'intégration future de la Hongrie à l'Union Européenne devrait encore renforcer cette inégalité, sensible dans de nombreux domaines dans ce pays.

En fonction de cela et dans le développement à long terme des transports, la priorité devrait être donnée aux points suivants :

- la construction de voies ferrées et d'autoroutes reliant directement les régions sans passer par la capitale, par exemple sous la forme de deux demi-cercles tracés au sud du pays ;

- le renforcement des dessertes quotidiennes aller et retour entre les villes, les villages et le chef-lieu du comitat, mais aussi avec les centres économiques les plus proches s'ils sont différents du chef-lieu. Le désenclavement est ainsi la clé du maintien d'une population rurale ;

- enfin un aéroport devrait être construit dans chaque grande région hongroise, moins dans le but de développer un trafic aérien intérieur assez faible dans un petit pays comme la Hongrie que de créer des liaisons avec les grands centres des pays voisins.

\section{BIBLIOGRAPHIE}

Deszényi M., Hernády F. (1967). - A magyar hajózás története (Une histoire des voies navigables en Hongrie), Muszaki K., Budapest, 458 p.

Eisenbahn- und Dampfschiffahrts- Buch für der Kaiserthum Österreich Tendler(1852). - Wien, 247 p.

Erdosi F. (1985). - Vasút és malomipar (voies ferrées et industrie agro-alimentaire), Vasút, vol. 35, $\mathrm{n}^{\circ} 8$, p. $10-11$.

Erdosi F. (1986). - Az egyközpontu vasúti fıvonalhálózat kialakítása a fıváros fejlesztése érdekében (Le réseau ferroviaire et le développement de la capitale), Közlekedéstudomány Szemle, vol. 36, n 9, p. 391-397.

Erdosi F. (1987a). - Monocentrikus tészerkezet és a közlekedési hálózat (Centralisme et réseau de transport), In :Csefko F. - dir., Állam, térkapcsolatok, demokrácia, Bihari Ottó emlékülés, MTA RKK JPTE, Pécs, p. 438-448.

Erdosi F. (1987b). - Területi érdekek és a vasúti közlekedés (Intérêts territoriaux et transports ferroviaires), Tér és Társadalom, n 1/1987, p. 47-65.

Erdosi F. (1987c). - Terüléti és ágazati érdekek a szocialista közlekedéshálózat alakulásában (Le gouvernement socialiste et les intérêts territoriaux et économiques), Ter és Társadalom, n 3/1987, p. $45-60$.

Erdosi F. (1988). - Budapest-központú vasúthálózat, monocentrikus térszerkezet (Le centralisme du réseau ferré sur la métropole de Budapest), Földrajzi Közlemények, nº 1-2/1988, p. 42-54. 
Erdosi F. (1989). - A vasutak egykori hatása az ország térszerkezetére és urbanizáció-jára (Le rôle passé du réseau ferroviaire sur l'alimentation des villes de province), Közlekedéstudomány Szemle, vol. 39, n 4, p. 175-183.

Erdosi F. (1990). - Politische und wirtschaftliche Motive des Eisenbahnbaues in Ungarn bis 1914, Österreichische Osthefte, $\mathrm{n}^{\circ} 1 / 1990$, p. 25-38.

Erdosi F. (1992). - The regional problems of Hungary's transport system, Geographical Review, $\mathrm{n}^{\circ} 116$, p. 25-38.

Erdosi F. (1996). - A Kárpát-medence közlekedési hálózatának alakulása és nemzetközi kapcsolódásai (Le réseau de transport du bassin des Carpates et les connexions internationales), In : Frisnyák S., A Kárpát-medence történeti Földrajza, Bessenyei György Tanárkézı Fliskola Földrajz Tanszek, Nyíregyháza, p. 225-238.

Erdosi F. (2000). - A közlekedés fejlidésének területpolitikai vonatkozásai és hatása a területkre és a településekre Magyarországon 1944-ig (Politique des transports, organisation spatiale et implantation des gares en Hongrie depuis 1944), OKTK MTA Regionális Kutatások Központja, Pécs, 358 p.

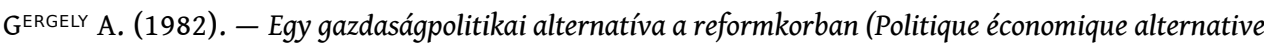
et réforme), Akadémiai Kiadó, Budapest, 135 p.

Sztankóczy Z. (1960). - Gazdasági és közlekedési földrajz (Géographie des transports et économie), Felsı oktatási Jegyzetelláto V., Budapest, 227 p.

Unyi B. (1989). - Vasuthálózatunk 1914-tıl napjainkig (Le réseau ferré de 1914 à aujourd'hui), KÖZDOK, Budapest, $363 \mathrm{p}$.

VÁSÁRHELYI B. (1959). - Közlekedésügy (À propos des transports), Tankönyvkiadó V., Budapest, 254 p.

\section{NOTES}

1. Correspond à la rive droite (ouest) du Danube, entre le fleuve et la frontière autrichienne.

2. Pest, Buda et Óbuda ont été réunies en 1872 pour former Budapest.

3. Sur la rive gauche (Est) du Danube.

4. La ville hongroise la plus peuplée au début du XIX ${ }^{\mathrm{e}}$ siècle était Debrecen avec 70000 habitants : voir à ce propos et dans ce numéro l'article de Szabó, Kiss et Lóki.

5. Correspond aujourd'hui à la Slovaquie.

6. Correspond aujourd'hui à l'Ukraine transcarpatique, connue aussi sous le nom de Ruthénie durant son intégration à la Tchécoslovaquie pendant l'Entre-deux-guerres.

7. Une carte des comitats, en 1913 et aujourd'hui, figure dans l'article de Hajdú, dans ce numéro.

\section{RÉSUMÉS}

Jusqu'au milieu du XIX ${ }^{\mathrm{e}}$ siècle, les faibles capacités de transport de marchandises, assurées essentiellement par des routes et chemins n'avaient pas induit d'importantes différences régionales en Hongrie. Ce n'est en fait qu'avec le développement sensible de la navigation fluviale que certaines régions et villes ont connu un développement plus significatif que d'autres. C'est 
ainsi que s'est formé un axe majeur d'urbanisation le long du Danube, rapidement centré sur Budapest, avec le développement d'un réseau ferré, puis d'un réseau routier et autoroutier en étoile autour de la capitale.

Cet article aborde donc, de façon chronologique, en ne quittant pas le cadre national hongrois, la mise en place progressive des réseaux de voies de communication en Hongrie, en insistant sur les mutations, occasionnées par leur construction, de l'organisation spatiale à l'échelle nationale et régionale.

Until the middle of the nineteenth century, poor good transport capacity essentially ensured that roads did not lead to important regional differences in Hungary. This does not mean that with the appreciable development of river navigation certain region and cities did not develop more significantly than others. Similarly a major axis of urbanisation formed along the Danube, rapidly centred on Budapest, with a development of a rail network and subsequently a road and motorway network focused on the capital.

This article then, first provides a chronology of the national Hungarian framework, then consider the types of communications network in Hungary and stresses the changes in spatial organisation at national and regional scales occasioned by their construction.

Bis zur Mitte des 19. Jahrhunderts hatten die schwachen Kapazitäten des Gütertransports, getragen vorwiegend durch Straßen und Wege, keine wesentlichen regionalen Unterschiede in Ungarn erzeugt. Erst mit dem spürbaren Ausbau der Flußschiffahrt haben manche Regionen und Städte eine bedeutsamere Entwicklung erfahren als andere. So hat sich eine Hauptachse der Urbanisierung längs der Donau gebildet - rasch auf Budapest zentriert - mit der Entwicklung eines Eisenbahn - dann eines Straßen - und Autobahnnetzes, sternförmig um die Hauptstadt.

Dieser Artikel befasst sich chronologisch und im Rahmen Ungarns mit der fortschreitenden Einrichtung der Verkehrsnetze in Ungarn und hebt dabei die konstruktionsbedingten Veränderungen der räumlichen Organisation im nationalen und regionalen Maßstab hervor.

INDEX

Keywords : Hungary, rail network, regional development, road network, transport Schlüsselwörter : Eisenbahnnetz, Regionalentwicklung, Straßennetz, Transport, Ungarn Mots-clés : développement régional, Hongrie, réseau ferré, réseau routier, transport

\section{AUTEURS}

\section{FERENC ERDOSI}

Centre d'études régionales - Académie Hongroise des Sciences - P.O box 199, 7601 Pécs, HONGRIE 